\title{
Uber die selektive Einwirkung verschiedener Faktoren auf die EMK und Respiration der Froschhaut.
}

\author{
Von \\ Naoyosi Endo. \\ (遠 藤 侣 義) \\ (Aus dem II. Institut für physiologie der Kaiserlichen Tohoku \\ Universität, Sendai, Vorstand : Prof. K. Motokawa.)
}

\section{Einleitung.}

Seit Lun ${ }^{1)}$ uns darauf aufmerksam machte, dass die Potentialbildung mit der Oxydation in ursächlichem Zusammenhang zu stehen scheint, hat sich eine Anzahl von Forschern, wie Willi a m u. Sheard ${ }^{2)}$, Taylor ${ }^{3}$, ' Francis s), Ponder u. Macleod ${ }^{5)}$ und $\mathrm{Huf(6)}$ mit der Frage beschäftigt, wie diese beiden Vorgänge miteinander verbunden sind. Sie konnten feststellen, dass atmungshemmende Bedingungen wie z. B. die Herabsetzung des partiellen Druckes des Sauerstoffs, atmungshemmende Gifte usw. auch eine Hemmung der EMK bewirken, aber dies spricht nicht ohne weiteres für die Lundsche Theorie, dass das Hautpotential ein Oxydo-Reduktionspotential sei.

Wird die Oxydation, welche die Lebensenergie versorgt, in oben erwähnter Weise gehemmt, so können alle Funktionen des Organismus mehr oder weniger stark beeinträchtigt werden, wobei die Funktion der Potentialbildung kaum eine Ausnahme bilden kann. Die Herabsetzung des Potentials durch Hemmung des Oxydationsprozesses weist nur darauf hin, dass das bioelektrische Potential eine Lebenserscheinung ist, kann jedoch keinen Aufschluss über den Entstehungsmechanismus des bioelektrischen Potentials geben.

Aus diesem Grunde führten Motokawa, Narita u. Endo einige Untersuchungen bei ganz intakt bleibender Atmung der Froschhaut aus, um die Beziehungen zwischen EMK und Respiration ausfindig zu machen, indem sie die Temperatur des Mediums innerhalb der physiologischen Grenzen veränderten. Sie haben bei diesen Untersuchungen festgestellt, dass eine lineare Beziehung zwischen der EMK und dem Logarithmus des $\mathrm{O}_{2}$-Verbrauchs besteht, und diese Beziehung theoretisch unter der Annahme erklärt, 
dass der Atmungsprozess ein konstantes Angebot der diffundierbaren Ionen schafft, um das Potential aufrecht zu erhalten. Dieselben Autoren ${ }^{8)}$ versuchten weiter die Beziehungen zwischen EMK und Respiration bei verschiedenen pH-Werten des Mediums aufzuklären, und stellten fest, dass sich die beiden auch unter diesen Bedingungen zueinander parallel verändern.

Diese beiden Untersuchungen scheinen ohne weiteres auf das Vorhandensein eines direkten kausalen Zusammenhangs zwischen EMK und Respiration hinzuweisen, aber folgendes Ergebnis von Ponder u. Macle od scheint sich kaum damit in Einklang bringen zu lassen: Saponin setzt die EMK beträchtlich herab, übt aber keinen Einfluss auf den $\mathrm{O}_{2}$-Verbrauch aus.

Wie kommen diese wenigstens scheinbar sich widersprechenden Ergebnisse zustande? Mit dieser Frage habe ich mich in vorliegender Arbeit in erster Linie beschäftigt. Konkreter ausgedrückt, versuchte ich in dieser Arbeit folgende Fragen $z u$ beantworten: 1. ob dieselben quantitativen Beziehungen, welche $z$ wischen der EMK und dem $\mathrm{O}_{2}$-Verbrauch nachgewiesen sind, auch unter unphysiologischen Bedingungen wie z. B. höheren Temperaturen von $40^{\circ}-60^{\circ} \mathrm{C}$ oder unter Einwirkung atmungshemmender KCNLösungen existieren, 2. ob Saponin auf EMK und $\mathrm{O}_{2}$-Verbrauch in der Tat selektiv einwirkt, wie Ponder u. Macleod behaupten, 3. unter welchen Bedingungen, seien es physikalische, chemische, oder physiko-chemische, EMK und $\mathrm{O}_{2}$-Verbrauch von einander gesondert beeinflusst werden.

\section{Versuchsmethodik.}

In vorliegender Untersuchung wurde die Haut des japanischen Wasserfrosches, Rana nigromaculata, als Versuchsmaterial verwendet. Die EMK wurde nach der Kompensationsmethode unter Benutzung eines Spiegelgalvanometers (Empfindlichkeit: $10^{-11} \mathrm{~A}$ ) als Nullinstrument gemessen, und der $\mathrm{O}_{2}$-Verbrauch durch die manometrische Methode mittels des Barcroft schen Apparates gemessen. Über Einzelheiten des Verfahrens sei auf die Arbeit von Endo ${ }^{97}$ verwiesen.

Die Versuchstemperatur war immer $25^{\circ} \mathrm{C} \pm 0,1$, und die Zusammensetzung der angewandten Ringerlösung $\mathrm{NaCl} 0,6 \%, \mathrm{KCl} 0,02 \%$ und $\mathrm{CaCl}_{2} 0,02 \%$,

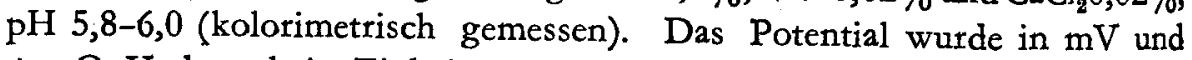
der $\mathrm{O}_{2}$-Verbrauch in Einheiten von $\mathrm{cmm} / \mathrm{g}$ (Trockengewicht) pro Stunde ausgedrückt.

\section{Versuchsergebnisse.}

Um die Fehlerbreite der Messungen zu bestimmen, wurde eine Anzahl von Leerversuchen ausgeführt, indem eine 1 Stunde lang gekochte tote Froschhaut als Versuchsmaterial angewendet wurde, und aus diesen Versuchen 
ergab sich, dass der mittlere Fehler der Manometermessungen nur $\pm 3,34 \%$ des $\mathrm{O}_{2}$-Verbrauchs normaler Haut derselben Grösse entsprach.

\section{A. Einfluss von Silbernitrat-Lösung.}

Nachdem das Potential einen stationären Zustand etreicht hatte, liessen wir eine 20\%ige Silbernitratlösung auf die Epithel- oder Serosafläche genau $1^{\prime \prime}, 2^{\prime \prime}, 3^{\prime \prime}, 5^{\prime \prime}, 30^{\prime \prime}$ und $180^{\prime \prime}$ lang einwirken. Dies geschah in folgender Weise : Ein Stück der sorgfältig abpräparierten Haut wurde an dem Ende eines durchlochten Reagenzglases von ca $6,0 \mathrm{~cm}$ Länge und vôn $1,2 \mathrm{~cm}$ Durchmesser mit Baumwollfäden derart befestigt, dass die Epithel- oder Serosafläche nach aussen gerichtet wurde, und der Serosa- oder Epithelfläche anhaftende Spuren der Ringerlösung wurden mit Fliesspapier sorgfältig abgesaugt. Das Präparat wurde darauf in die Silbernitratlösung getaucht und nach einer bestimmten Zeit aus der Lösung herausgenommen, um das der Haut anhaftende Silbernitrat als Niederschlag von $\mathrm{AgCl}$ mit einer grossen Menge Ringerlösung zu beseitigen. Sogleich nach der Anwendung des Silbernitrates auf die Epithelfäche, zeigte sich eine starke Herabsetzung der EMK, welche bai der Ringer-Ringer-Ableitung gemessen wurde, und die so herabgesetzte EMK blieb lange Zeit unverändert, ohne dass sie Zeichen der Erholung oder der weiteren Abnahme zeigte. Dieses Verhalten ist umso merkwürdiger, als sich die EMK nach Einwirkung anderer Lösungen wie z.B. KCN, Saponin usw. mit der Zeit verändert, wie unten exläutert werden wird.

Die Hemmungsgrösse der EMK betrug 100\% bei einer Einwirkungsdauer des Silbernitrates von $1^{\prime \prime}$ und $2^{\prime \prime}$, war aber unter Umständen noch geringer. Bei einer Einwirkungsdauer von $3^{\prime \prime}$ (16 Fälle), $5^{\prime \prime}$ (8 Fälle) und $30^{\prime \prime}$ (4 Fälle) betrug die Hemmung des Potentials immer $100 \%$, aber der $\mathrm{O}_{2}$-Verbrauch blieb bei einer Einwirkungsdauer von $3^{\prime \prime}$ und 5 " merkwürdigerweise fast' intakt. Er wurde durch Silbernittat-Einwirkung von $30^{\prime \prime}$ bis

Tabelle I (a).

Bei der Anwendung der 20\%igen Silbernitratlösung auf die Epithelfläche.

\begin{tabular}{c|c|c}
\hline $\begin{array}{c}\text { Einwirkungsdauer } \\
\text { in Sek. }\end{array}$ & $\begin{array}{c}\text { EMK in Proz. derselben } \\
\text { vor d. Anwendung }\end{array}$ & $\begin{array}{c}\mathrm{O}_{2} \text {-Verbrauch in Proz. } \\
\text { d. Kontrolle }\end{array}$ \\
\hline 3 & 0 & 93,0 \\
\hline 5 & 0 & 99,9 \\
\hline 30 & 0 & 41,1 \\
\hline 180 & 0 & 17,1
\end{tabular}


auf $41,1 \%$ (6 Fälle), und bei einer Einwirkungsdauer von $180^{\prime \prime}$ bis auf 7,3\% (5 Fälle) herabgesetzt (Tab. I (a)).

Tabelle I (b).

Bei der Anwendung der 20\%igen Silbernitratlösung auf die Serosafläche.

\begin{tabular}{c|c|c}
\hline $\begin{array}{c}\text { Einwirkungsdauer } \\
\text { in Sek. }\end{array}$ & $\begin{array}{c}\text { EMK in Proz. derselben } \\
\text { vor d. Anwendung }\end{array}$ & $\begin{array}{c}\mathrm{O}_{\mathbf{2}} \text {-Verbrauch in Proz. } \\
\text { d. Kontrolle }\end{array}$ \\
\hline 3 & 24,2 & 119,0 \\
\hline 5 & 16,3 & 99,8 \\
\hline 30 & 0 & 25,3 \\
\hline 180 & 0 & 16,3 \\
\hline
\end{tabular}

Bei Anwendung der 20\%igen Silbernitratlösung auf die Serosafläche für 3" und 5", nahm wie aus Tab. II (b) ersichtlich wird, die EMK in 90 Minuten bis auf $24,3 \%$ resp. $16,3 \%$ ab, und bei Anwendung einer Lösung gleicher Konzentration für $30^{\prime \prime}$ und $180^{\prime \prime}$ wurde sie sogleich bis auf Null herabgesetzt. Der $\mathrm{O}_{2}$-Verbrauch zeigte bei der Anwendung der 20\%igen Silbernitratlösung für 3 " eine geringe Zunahme ( 6 Fälle), aber bei 5 Sekunden langer Einwirkung keine Veränderung (5 Fälle) und wurde bei längerer Einwirkung wie $30^{\prime \prime}$ und $180^{\prime \prime}$ bis auf $25,3 \%$ (4 Fälle) resp. $16,3 \%$ ( 3 Fälle) herabgesetzt.

Es liegt nahe, dass Ag-Ionen bei der Anwendung der 20\%igen Silbernitratlösung auf die Epithelfäche durch Diffusion an die Epidermisschichten gelangen, wie an Schnittpräparaten nachgewiesen wurde, und dass sie die EMK, welche wahrscheinlich auf die Epidermisseite der Haut beschränkt lokalisiert ist, bis auf Null herabsetzen. Im Gegensatz dazu wird der $\mathrm{O}_{2}-$ Verbrauch durch das Eindringen des Silbernitrates in die Epidermisschichten nicht so stark beeinflusst, denn das $\mathrm{NaCl}$ im Gewebe hemmt die Diffusion des Silbernitrates in die tieferen Schichten der Haut.

Da die Wirkung der 20\%igen Silbernitratlösung wie oben erwähnt, auf die angewandte Fläche beschränkt ist, kann man schliessen, dass die Potentialsprünge an den Epidermisschichten existieren, denn das Potential wird nur bei Einwirkung des Silbernitrats auf die Epithelfläche stark beeinträchtigt. Bei der Anwendung der 20\%igen Silbernitratlösung auf die Serosafläche ist die Herabsetzung der EMK dagegen nur geringfügig, denn Ag-Ionen können bei einer so kurzen Anwendungsdauer wie beider von 3" oder 5" nicht an die Epidermisschichten gelangen, die ja den Sitz der EMK darstellen.

Die Tatsache, dass der $\mathrm{O}_{2}$-Verbrauch bei derselben Einwirkungsdauer 
des Agens auf die Epithelfläche weniger stark beeinträchtigt wird, als bei dessen Anwendung auf die Serosafäche, lässt sich darauf zurückführen, dass erstere im allgemeinen gegen alle Substanzen widerstandsfähiger als letztere ist.

Aus diesen Untersuchungen kann man schliessen, dass die selektive Wirkung des Agens auf die EMK und den $\mathrm{O}_{2}$-Verbrauch auf dem Lokalisationsunterschied-EMK in den Epidermisschichten einerseits und $\mathrm{O}_{2}-\mathrm{Ver}$ brauch in allen Hautschichten andererseits - beruht.

B. Einfluss mechanischer Reize.

Die Froschhaut wurde durch die dorsale und ventrale Mittellinie in eine rechte und eine linke Hälfte halbiert, von denen eine Kontrollversuchen diente. Um nur die oberflächlichen Schichten der Haut mechanisch zu schädigen, wurde das Hautstück am Rande fixiert und die Epithel- oder Serosafäche mit einer Rasierklinge unter einem Neigungswinkel von etwa $45^{\circ}$ stark gestrichen. Dieser mechanische Eingriff war so wirksam, dass deutliche Petechien hervorgerufen wurden, wenn dieselbe Prozedur auf dem Handrücken des Menschen ausgeführt wurde.

Die EMK wurde beim Abschaben der Epithelfäche durch diese Prozedur sofort bis auf Null herabgesetzt, wie es auch bei den du Bois-Reymond-schen Untersuchungen (1865) der Fall war. Das Verschwinden der EMK ist keine vorübergehende Erscheinung, denn die EMK zeigte kein Zeichen der Erholung. Im Gegensatz zu dieser starken Wirkung auf die EMK wurde der $\mathrm{O}_{2}$-Verbrauch durch dieselbe Prozedur nur bis auf 76,5\% (6 Fälle) herabgesetzt. Aber das Verhältnis war beim Abschaben der Serosafläche ganz umgekehrt; die EMK betrug bei diesem Fall im Durchschnitt von 6 Fällen 92,7\%. In Anbetracht dessen, dass der Unterschied des Potentials zwischen den beiden symmetrischen Hauthälften einige Prozente betragen kann, kann man aus diesem Ergebnis schliessen, dass die Schädigung der Serosafläche auf die EMK gar keinen Einfluss ausübt. Im Gegensatz dazu wurde der $\mathrm{O}_{2}$-Verbracuh durch diese Prozedur stärker beeinträchtigt,

Tabelle II.

Einfluss des mechanischen Reizes.

(Abschaben mit einer Rasierklinge)

\begin{tabular}{l|c|c}
\hline Abgeschabte Fläche & $\begin{array}{c}\text { EMK in Proz. derselben } \\
\text { vor d. Anwendung }\end{array}$ & $\begin{array}{c}\mathrm{O}_{2} \text {-Verbrauch in Proz. } \\
\text { d. Kontrolle }\end{array}$ \\
\hline Epithelfäche & 0 & 76,5 \\
\hline Serosafläche & 100 & 75,2
\end{tabular}


z.B. betrug nach dem Abschaben der Serosafläche der $\mathrm{O}_{2}$-Verbrauch 75,2\% (6 Fälle) des Wertes vor dem Eingriff.

Tabelle III.

Einfluss der Saponin-Ringerlösung.

\begin{tabular}{c|c|c}
\hline $\begin{array}{c}\text { Konzentration des } \\
\text { Saponins in \% }\end{array}$ & $\begin{array}{c}\text { EMK in Proz. derselben } \\
\text { vor d. Anwendung }\end{array}$ & $\begin{array}{c}\mathbf{O}_{2} \text {-Verbrauch in Proz. } \\
\text { d. Kontrolle }\end{array}$ \\
\hline 0,05 & 0 & $\mathbf{7 4 , 0}$ \\
\hline 0,5 & 0 & $.69,1$
\end{tabular}

C. Einfluss des Saponins.

Bei der Ponder u. Macleod ${ }^{5)}$ schen Untersuchung benutzten die Verfasser eine kleine Menge Saponin-Lösung (nur $1 \mathrm{ccm}$ ), aber die Wirkung des Saponins kann bei einer so kleinen Menge durch das Sektet aus der Haut und dgl. gemildert werden, so dass EMK und $\mathrm{O}_{2}$-Verbrauch an der mit einer $0,05 \%$ oder $0,5 \%$ Saponin-Ringerlösung von etwa $15 \sim 20$ $\mathrm{ccm}$ genügend lang, nämlich 5 Stunden lang vorbehandelten Haut gemessen wurde.

Die Messung der ersteren wurde dann in der Ringer- und die des letzteren in der $0,05 \%$ oder $0,5 \%$ Saponin-Ringerlösung ausgeführt.

Wie in Tab. III ersichtlich, wurde die EMK nach der Anwendung der beiden Saponin-Ringerlösungen gleichfalls bis auf Null herabgesetzt, aber der $\mathrm{O}_{2}$-Verbrauch betrug in der $0,05 \%$ Saponin-Ringerlbsung $74,0 \%$ und in der $0,5 \%$ Saponin-Ringerlösung $69,1 \%$. Ponder u. Macleod bemerkten zuweilen bei. Anwendung der Saponin-Lösung eine geringe Zunahme des $\mathrm{O}_{2}$-Verbrauchs, die aber wahrscheinlich auf Messungsfehlern beruht, wie Ponder u. Macleod selbst zugaben, denn nach meinen sorgfältigen Untersuchungen wirkt dieses auf das Protoplasma giftig wirkende Mittel immer auf die EMK und den $\mathrm{O}_{2}$-Verbrauch hemmend, aber in von einander verschiedener Weise wie unten gezeigt werden wird. Nach Ponder u. Macleod wurde die EMK in einer 1\%igen Saponin-Lösung um $85 \%$ und in einer $2 \%$ igen Saponin-Lösung um 100\% gehemmt. Viel stärker war die Hemmung bei meinen Untersuchungen, z.B. wurde die EMK schon in der 0,05\% SaponinRingerlösung um $100 \%$ gehemmt, während der $\mathrm{O}_{2}$-Verbrauch nur um $26 \%$ herabgesetzt wurde. Dieser Unterschied beruht vielleicht darauf; dass die EMK, welche in den Epidermisschichten lokalisiert ist, durch die Oberflächenaktivität des Saponins stärker beeinflusst wird, als $\operatorname{der} \mathrm{O}_{2}$-Verbrauch, an welchem alle Hautzellen teilnehmen. 
D. Einfluss der Temperatur.

Ein stationärer Zustand der EMK wurde bei der Ringer-Ringer-Ableitung gewöhnlich in 1-4 Stunden erreicht, und die Verändetung der EMK blieb dann innerhalb $1 \mathrm{mV}$ in $30-40$ Minuten. Nachdem die Haut diesen Zustand erreicht hatte, wurde sie für 30 Minuten in Ringerlösungen von $30^{\circ} \mathrm{C}$, $35^{\circ} \mathrm{C}, 40^{\circ} \mathrm{C}, 43^{\circ} \mathrm{C}, 45^{\circ} \mathrm{C}, 46^{\circ} \mathrm{C}, 50^{\circ} \mathrm{C}$ und $60^{\circ} \mathrm{C}$ getaucht, und datauf wurden die EMK und der $\mathrm{O}_{2}$-Verbrauch in einem Thermostaten bei $25^{\circ} \mathrm{C} \pm 0,1$ gemessen. Bei der Messung des $\mathrm{O}_{2}-$ Verbrauchs wurde die rechte symmetrische Hauthälfte als Kontrolle benutzt, und die linke wurde der erwähnten Tempetatur für 30 Minuten ausgesetzt. Der $\mathrm{O}_{2}$-Verbrauch der geschädigten Haut wurde in Prozenten des Kontrollversuches ausgedrückt.

Tabelle IV (a).

Einfluss der Temperatur.

\begin{tabular}{c|c|c}
\hline $\begin{array}{c}\text { Wassertemperatur und } \\
\text { Einwirkungsdauer }\end{array}$ & $\begin{array}{c}\text { EMK in Proz. derselben } \\
\text { vor d. Anwendung }\end{array}$ & $\begin{array}{c}\text { O-Verbrauch in Proz. } \\
\text { d. Kontrolle }\end{array}$ \\
\hline $30^{\circ} \mathrm{C} 30^{\prime}$ & 100,0 & 105,3 \\
\hline $35^{\circ} \mathrm{C} 30^{\prime}$ & 73,5 & 90,5 \\
\hline $40^{\circ} \mathrm{C} 30^{\prime}$ & 97,5 & 93,5 \\
\hline $43^{\circ} \mathrm{C} 30^{\prime}$ & 45,0 & - \\
\hline $45^{\circ} \mathrm{C} 30^{\prime}$ & 17,0 & 53,0 \\
\hline $46^{\circ} \mathrm{C} 30^{\prime}$ & 0 & - \\
\hline $50^{\circ} \mathrm{C} 30^{\prime}$ & 0 & 36,6 \\
\hline $55^{\circ} \mathrm{C} 30^{\prime}$ & 0 & 10,5 \\
\hline $60^{\circ} \mathrm{C} 30^{\prime}$ & 0 & 0 \\
\hline
\end{tabular}

Tabelle IV (b).

Einfluss der Einwirkungsdauer.

\begin{tabular}{c|c|c}
\hline $\begin{array}{c}\text { Wassertemperatur und } \\
\text { Einwirkungsdauer }\end{array}$ & $\begin{array}{c}\text { EMK in Proz. derselben } \\
\text { vor d. Anwendung }\end{array}$ & $\begin{array}{c}\mathrm{O}_{2} \text {-Verbtauch in Proz. } \\
\text { d. Kontrolle }\end{array}$ \\
\hline $440^{\circ} \mathrm{C} 60^{\circ}$ & 100,0 & 91,8 \\
\hline $640^{\circ} \mathrm{C} 120^{\circ}$ & 5,5 & 81,8
\end{tabular}

Wie aus der Tab. IV (a) und Abb. 1 ersichtlich ist, wurde die EMK bei einer Einwirkung der Temperatur von $20^{\circ} \mathrm{C}$ für 30 Minuten gar nicht beeinflusst (12 Fälle). Sie betrug bei der Temperatur von $35^{\circ} \mathrm{C} 73,5 \%$ (16 Fälle), bei $40^{\circ} \mathrm{C} 27 ; 5 \%$ (18. Fälle), und nahm bei weiterer Temperatursteigerung bis 
zu $45^{\circ} \mathrm{C}$ fast linear ab, um bei $46^{\circ} \mathrm{C}$ zu verschwinden. Der $\mathrm{O}_{2}$-Verbrauch nahm dagegen bei $30^{\circ} \mathrm{C}$ bis auf $105,3 \%$ (5 Fälle) zu, blieb bei $40^{\circ} \mathrm{C}$ unverändert, betrug bei $45^{\circ} \mathrm{C} 53,0 \%$ ( 5 Fälle) und nahm bei weiterer Steigerung der Temperatur fast linear $\mathrm{ab}$, um bei $60^{\circ} \mathrm{C}$ ganz zu verschwinden. Die Respiration der Froshchaut blieb also bis zur Anwendung einer so hohen Temperatur wie $55^{\circ} \mathrm{C}$ erhalten, während die EMK schon bei $46^{\circ} \mathrm{C}$ bis auf Null herabgesetzt wurde.

Die Tatsache, dass die EMK bei der 30 Minuten langen Anwendung von Temperauren über $40^{\circ} \mathrm{C}$ bis auf Null herabgesetzt wird, während die Respiration noch fortgesetzt wird, legt die Annahme nahe, dass der Potentialbildung unversehrte Zell- und Gewebestrukturen und Funktionen zugrunde liegen müssen, die sich nur bei austeichender Energieversorgung durch Oxydationsprozesse aufrecht erhalten lassen.

Die Hautatmung wurde bei 30 Minuten langer Anwendung von Temperaturen von $35^{\circ} \mathrm{C}$ und $45^{\circ} \mathrm{C}$ nicht beträchtlich gestört, aber bei gleich langer Anwendung einer Temperatur von $45^{\circ} \mathrm{C}$ sehr stark herabgesetzt. Diese Tatsache ist so zu deuten, dass die mit der Respiration in Beziehung stehenden wichtigen Fermente zwischen $40^{\circ}-45^{\circ} \mathrm{C}$ zerstört werden. Der Anstieg des $\mathrm{O}_{2}$-Verbrauchs bei der 30 Minuten langen Anwendung einer Temperatur von $30^{\circ} \mathrm{C}$ beruht darauf, dass diese Temperatur innerhalb der physiologischen Grenzen bleibt, in denen der $\mathrm{O}_{2}$-Verbrauch umso grösser ist, je höher die Temperatur ist.

Das Dreieck NOP in Abb. 1 entspricht dem von der EMK völlig unabhängigen $\mathrm{O}_{2}$-Verbrauch. Es ist hervorzuheben, dass die EMK bəi einer Temperatur von $46^{\circ} \mathrm{C}$ Null ist, während vom $\mathrm{O}_{2}$-Verbrauchnoch 46 $\%$ vorhanden sind. Dasselbe Verhältnis zeigt sich auch bei der Anwendung der Abb. 1. EM K und $\mathrm{O}_{2}$-Verbrauch der Froschhaut nach der 30 Minuten langen Einwirkung verschiedener Temperaturen:

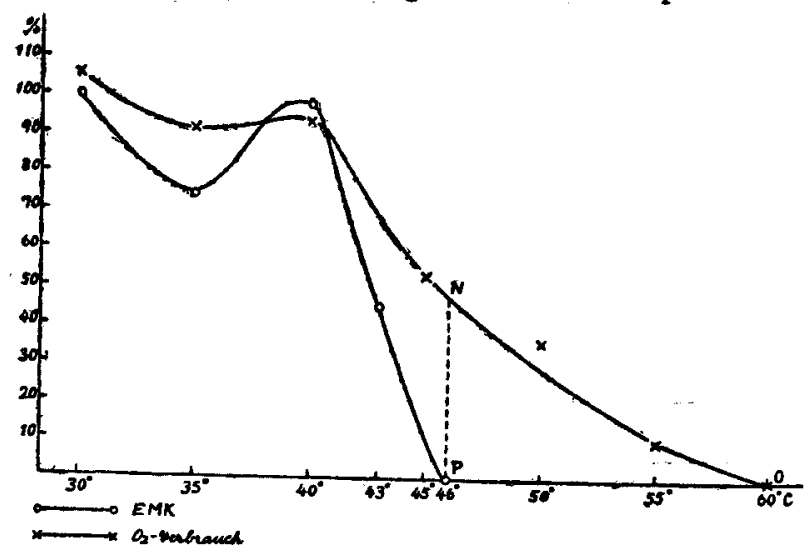
M/750 KCN-Ringerlösung, worauf ich unten noch zurückkommen werde.

Es wurde weiter die Abhängigkeit von der Wirkungsdauer bei ein und derselben Temperatur, nämliche $40^{\circ} \mathrm{C}$ untersucht. Das Ergebnis ist in Tab. 
IV(b) dargestellt. Wie daraus hervorgeht, wurde die EMK durch 60 Minuten lange Anwendung dieser Temperatur in 11 Fällen kaum beeinflusst, und dabei betrug der $\mathrm{O}_{2}$-Verbrauch $91,8 \%$ ( 6 Fälle), und nach 120 Minuten langer Anwendung betrug das Potential nur 5,5\% (6 Fälle), während der $\mathrm{O}_{2}$-Verbrauch $81,8 \%$ bitrug ( 5 Fälle).

Aus diesem Ergebnis geht ohne weiteres hervor, dass der $\mathrm{O}_{2}$-Verbrauch bei den Anwendung einer Temperatur von $40^{\circ} \mathrm{C}$ etwas gehemmt wird, aber dass solche Hemmung keinen Einfluss auf die EMK hat. Durch die 120 Minuten lange Einwirkung wurde die EMK sehr stark herabgesetzt, und mit dieser starken Abnahme der EMK ging eine geringe Reduktion des $\mathrm{O}_{2}$ Verbrauches, nämlich eine solche von $20 \%$ einher. Diese Tatsache deutet darauf hin, dass das, was für das Potential massgebend ist, nicht der gesamte $\mathrm{O}_{\mathbf{2}}$-Verbrauch, sondern ein Bruchteil desselben sein muss.

\section{E. Einfluss der KCN-Einwirkung.}

Die KCN-Einwirkung auf die EMK ist von $\mathrm{Lund}{ }^{10)}, \mathrm{Hu} \mathrm{f}^{13) 14)}$ und besonders von $\mathrm{Narita}^{11)}$ in ausführlicher Weise untersucht worden. In meiner Arbeit wurde die $\mathrm{KCN}$-Wirkung auf die EMK und den $\mathrm{O}_{2}$-Verbrauch derart untersucht, dass die Haut, welche in einer $0,05 \%$ igen oder $0,5 \%$ igen $\mathrm{KCN}$ Ringerlösung von 15-20 ccm 5 Stunden aufbewahrt worden war, auf die EMK hin in Ringerlösung und auf den $\mathrm{O}_{2}$-Verbaruch hin in $0,05 \%$ oder $0,5 \%$ KCN-Ringerlösung gemessen wurde.

Wie in Tab. V dargestellt ist, wurde die EMK 30 Minuten nach der Anwendung der $\mathrm{M} / 50-\mathrm{KCN}$-Ringerlösung und 90 Minuten nach det Anwendung der $\mathrm{M} / 200$-, $\mathrm{M} / 500$ - und $\mathrm{M} / 750-\mathrm{KCN}$-Ringerlösungen bis auf Null herabgesetzt, darauf hinweisend, dass die EMK mit der Zunahme der KCNKonzentration schneller herabgesetzt wird. Bei der Anwendung der M/50-,

Tabelle V.

Einfluss der KCN-Ringerlösung.

\begin{tabular}{c|c|c}
\hline $\begin{array}{c}\text { Konzentration d. KCN } \\
\text { in Ringer }\end{array}$ & $\begin{array}{c}\text { EMK in Proz. derselben } \\
\text { vor d. Anwendung }\end{array}$ & $\begin{array}{c}\mathrm{O}_{2} \text {-Verbrauch in Proz. } \\
\text { d. Kontrolle }\end{array}$ \\
\hline $\mathrm{M} / 50$ & 0 & 42,0 \\
\hline $\mathrm{M} / 200$ & 0 & 56,7 \\
\hline $\mathrm{M} / 500$ & 0 & 41,7 \\
\hline $\mathrm{M} / 750$ & 0 & 43,0 \\
\hline $\mathrm{M} / 1,000$ & 5,4 & 71,5 \\
\hline $\mathrm{M} / 10,000$ & 14,8 & 72,1 \\
\hline
\end{tabular}


Tabelle VI.

Einfluss der $\mathrm{M} / 1,000 \mathrm{KCN}$-Ringerlösung auf den $\mathrm{O}_{2}$ Verbrauch (cmm/g. Std. Trockengewicht).

\begin{tabular}{c|c|c}
\hline Kontrolle & $\begin{array}{c}\mathrm{O}_{2} \text {-Verbrauch nach d. } \\
\text { Anwendung }\end{array}$ & $\%$ \\
\hline 1231,93 & 907,98 & 73,3 \\
\hline 1365,17 & 967,60 & 70,9 \\
\hline 708,01 & 465,50 & 65,6 \\
\hline 841,67 & 472,83 & 56,2 \\
\hline 656,96 & 499,62 & 76,0 \\
\hline 1238,55 & 1007,18 & 81,3 \\
\hline
\end{tabular}

$\mathrm{M} / 200-, \mathrm{M} / 500$ - und $\mathrm{M} / 750-\mathrm{KCN}-$ Ringerlösung wurde $\operatorname{der} \mathrm{O}_{2}$-Verbrauch in gleicher Weise um 60\% gehemmt und bei der Anwendung der M/1,000- und M/10,000-KCN-Ringerlösung etwa um 30\% gehemmt (Tab. VI).

Es ist schon bekannt, dass die EMK durch hohe Konzentrationen von KCN-Lösungen bis auf Null herabgesetzt wird, aber die Ergebnisse über den $\mathrm{O}_{2}$-Verbrauch der Froschhaut bei KCN-Wirkung sind von Forscher zu Forscher von einander sehr verschieden; nach $\mathrm{Adol} \mathrm{ph}^{12)}$ wurde der $\mathrm{O}_{2}$ Verbrauch in $0,01 \mathrm{M}-\mathrm{KCN}$-Lösung um $50 \%$, aber in $0,002 \mathrm{M}$ - und $0,001 \mathrm{M}$ $\mathrm{KCN}$-Lösung gar nicht herabgesetzt, und nach $\mathrm{H}$ u f ${ }^{\text {13)14) }}$ wurde der $\mathrm{O}_{2}$-Vefbrauch an der Rückenhaut, der mittels des Warburg schen Manometers gemessen wurde, in 0,001M-KCN-Lösung um 45,5\% (1 Fall) und in 0,002M$\mathrm{KCN}$-Lösung um $68,8 \%$ ( 3 Fälle) gehemmt. Falls der $\mathrm{O}_{2}$-Verbrauch mittels des Winkler schen Apparates gemessen wurde, wurde er in 0,001M-KCNLösung bis auf $17,5 \%$ herabgesetzt. Diese Unterschiede scheinen nicht nur von dem jahreszeitlichen Zustand des Frosches, sondern von der Airt und Weise der Anwendung.der KCN-Lösungen abhạ̈ngig zu sein. Auf jeden Fall ist sehr merkwürdig, dass die EMK durch die KCN-Einwirkung bis auf Null herabgesetzt würde, während $\operatorname{der} \mathrm{O}_{2}$ - Verbrauch nicht völlig gehemmt warde.

\section{Diskussion der Ergebnisse.}

Aus den erwähnten Untersuchungen geht hervor, dass sich die EMK und der $\mathrm{O}_{2}$-Verbrauch unter Umständen nicht immer parallel zueinander verändern; die EMK witd je nach den Bedingungen bis auf Null herabgesetzt, während der $\mathrm{O}_{2}$-Verbrauch dabei nicht stark beeinflusst wird, und unter Umständen fällt das Verhältnis ganz umgekehrt aus. 
Das erstere Verhalten zeigte sich, wenn

i) eine $20 \%$ Silbernitratlösung auf die Epithelfäche angewendet wurde,

ii) die Epithelfäche mit der Rasierklinge abgeschabt wurde und

iii) die Haut mit einer 0,05\% Saponin-Ringerlösung behandelt wurde.

Das letztere Verhalten wurde bemerkt, wenn

i) die Serosafläche mit der Rasierklinge abgeschabt wurde und auch ii) wenn die Haut 60 Minuten lang in Ringerlösung von $40^{\circ} \mathrm{C}$ getaucht wurde.

Diese Tatsachen könnten zu dem Schluss führen, dass keine Beziehungen zwischen der EMK und dem $\mathrm{O}_{2}$-Verbrauch existieren, aber man kann in Anbetracht der folgenden Umstände keineswegs die innigen Beziehungen zwischen den beiden negieren. Motok a w $\mathbf{a}^{15}$ hat aus verschiedenen Gründen geschlossen, dass Potentialsprünge der Froschhaut in den lebendigen Schichten auf der Epidermis-Seite der Haut, besonders im Stratum germinativum, existieren. Daher scheint es fast selbstverständlich, dass die EMK sich vermindert oder ganz und gar verschwindet, wenn die Epidermisschichten durch eine $20 \%$ ige Silbernitratlösung geätzt werden, oder wenn sie mechanisch abgeschabt werden. Aber die Zellen, die Sauerstoff verbrauchen, sind nicht nur auf die Epidermisschichten beschränkt, sondern in allen Schichten der Haut verteilt, so dass sich der $\mathrm{O}_{2}$-Verbrauch durch die erwähnten Prozeduren niemals bis auf Null herabsetzen lässt. Die Tatsache, dass Saponin selektiv nur auf die EMK stark hemmend wirkt, ist aus seiner bekannten Oberflächenaktivität leicht verständlich.

Im zweiten Falle, in welchem nur der $\mathrm{O}_{2}$-Verbrauch selektiv beeinflusst wird, bleibt die Epidermis-Seite der Haut verhältnismässig intakt, z.B. wenn nur die Hypoderm-Fläche abgeschabt wird. Durch dieses Verfahren werden die Zellen in der Cutis etwas zerstört, so dass der $\mathrm{O}_{2}$-Verbrauch mehr oder weniger stark beeinträchtigt wird, während die EMK fast unverändert bleibt.

Wie aus dem erwähnten hervorgeht, ist die Widerstandskraft der Hautatmung gegen die $\mathrm{KCN}$-Wirkung und hohe Temperaturen im allgemeinen grösser als die des Hautpotentials, was sich aus dem Mechanismus der Potentialbildung ohne Schweierigkeiten verstehen lässt. Es unterliegt keinem Zweifel, dass physiko-chemische Zustände des Gewebes, insbesondere die Verteilung der Ionen an der Grenzfläche für das Potential massgebend sind, und dass die Grösse der nach aussen ableitbaren EMK je nach dem Nebenschluss im Gewebe verschieden ausfällt, der seinerseits mit der Art und Weise der Gewebsstruktur eng zusammenhängt. Wenn das Gewebe durch die Wirkung verschiedener Agentien und hoher Temperaturen aufgelockert wird, so kann die EMK infolge vermehrten „Shunt"-Effekts stark reduziert werden, ohne dass die Funktion der Atmungsfermente gestört wird. Es ist 
denkbar, dass die Auflockerung des Gewebes auf die Hautatmung vielmehr fördernd wirkt, weil der Sauerstoff dabei leichter zu den Atmungsfermenten gelangen kann. Um die EMK verschwinden zu lassen, ist es aus dem etwähnten Gründen nicht immer nötig, die Oxydation zumStillstand zu bringen; meine Untersuchungen zeigen in der Tat, dass eine Reduktion der Atmung um einige Zehntel des normalen $\mathrm{O}_{2}$-Verbrauchs ausreicht, um die EMK bis auf Null herabzusetzen.

Wenn die EMK durch die Wirkung einer bestimmten hohen Temperatur und einer KCN-Lösung bestimmter Konzentration $\epsilon$ ben verschwand, so verbrauchte die Haut noch $46 \%$ resp. $43 \%$ der normalen Sauerstoffmenge. Diese Übereinstimmung ist umso bemerkenswerter, als die beiden Eingriffe von einander sehr verschiedener Natur sind. Es liegt nahe, dass der „Shunt"-Effekt durch Zustandsveränderungen des Gewebes infolge des reduzierten $\mathrm{O}_{2}$-Verbrauchs verstärkt wurde, oder dass die von der Oxydation direkt oder indirekt abhängige Bildung der am Potential teilnehmenden Ionen gestört wurde. Jedenfalls lässt sich das erwähnte voneinander abweichende Verhalten der EMK und des $\mathrm{O}_{2}$-Verbrauchs unter abnormen Bedingungen auf diese Weise auf Verschiedenheiten der hauptsächlich. daran teilnehmenden Gewebeschichten einerseits und auf Unterschiede der Ejgenschaften beider Erscheinungen andererseits zurückführen, so dass man aus Ergebnissen unter unphysiologische Bedingungen nicht ohne weiteres den ursächlichen Zusammenhang ausschliessen darf, welcher nach den Untersuchungen von Motokawa, Narita u. Endo sehr wahrscheinlich zwischen beiden Erscheinungen besteht. So innig die Beziehungen auch sein mögen, so darf man das Hautpotential doch nicht als Oxydo-Reduktionspotential betrachten, worauf Beutner u. Lozne ${ }^{16)}$, Beutene $\mathbf{r}^{17)}$, Stern ${ }^{18)}$


$\mathrm{Na}$ rit a u. Endo annahmen, sind der $\mathrm{O}_{2}$-Verbrauch und die EMK vielleicht durch die Erhaltung des Kolloidalzustandes und die oxydative Versorgung der mit der Potentialbildung in Beziehung stehenden spezifischen Ionen miteinander verbunden.

\section{zusammenfassung.}

Um den Zusammenhang zwischen der EMK und der Respiration der Froschhaut unter verschiedenen Bedingungen $\mathrm{zu}$ erforschen, wurden die EMK nach der Kompensationsmethode und der $\mathrm{O}_{2}$-Verbrauch mittels des Barcroftschen Manometers parallel zueinander untersucht.

1) Wird die Epithelfäche mit einer $20 \%$ igen Silbernitratlösung für kurze Zsit wie 1"-2" verätzt; so wird die EMK beträchtlich oder bis auf 
Null herabgesetzt, aber der $\mathrm{O}_{2}$-Verbrauch wird durch diese Prozedur kaum beeinflusst. Wird dieselbe Lösung auf die Serosafläche angewendet, so wird die EMK weniger stark beeinträchtigt als der $\mathrm{O}_{2}$-Verbrauch.

2) 'Wird die Epithelfläche mit der Rasierklinge abgeschabt, so wird die EMK sofort bis auf Null herabgesetzt, aber der $\mathrm{O}_{2}$-Verbrauch wird kaum beeinflusst, und beim Abschaben der Serosafläche ist das Resultat im grossen und ganzen umgekehrt.

3) $0,05 \%$ ige und $0,5 \%$ ige Saponin-Ringerlösung wirken auf die EMK und den $\mathrm{O}_{2}$-Verbrauch hemmend, aber die Hemmungsgrösse ist bei der EMK viel grösser als beim $\mathrm{O}_{2}$-Verbrauch. Aus diesen Tatsachen geht ohne weiteres hervor, dass das Hautpotential an den Epidermisschichten lokalisiert ist.

4) Wird die Haut für 30 Minuten einer so hohen Temperatur wie über $30^{\circ} \mathrm{C}$ ausgesetzt, so werden die EMK und der $\mathrm{O}_{2}$-Verbrauch herabgesctzt, und zwar in verschiedenem Masse : Bei einer 30 Minuten langen Anwendung einer Temperatur von: $46^{\circ} \mathrm{C}$ wird die EMK bis auf Null herabgesetzt, während noch $46 \%$ des $\mathrm{O}_{2}$-Verbrauches bestehen bleiben.

5) Die $\mathrm{M} / 50-\mathrm{KCN}$-Ringerlösung kann selbst bei genügend langer Wirkungsdauer wie z.B. über 5 Stunden den $\mathrm{O}_{2}$-Verbrauch nicht völlig hemmen. Die M/750-KCN-Ringerlösung kann die EMK auf Null bringen, aber den $\mathrm{O}_{2}$-Verbrauch nur um $57 \%$ vermindern.

4) und 5) zeigen, dass ein bestimmter Bruchteil, wie etwa $40 \%$ des normalen $\mathrm{O}_{2}$-Verbrauchs für die Erhaltung des Hautpotentials notwendig ist.

Am Schluss spreche ich Herrn Prof. K. Motokaw a für seine freundliche Leitung und Unterstützung bei dieser Arbeit meinen herzlichsten Dank aus, und auch Dr. T. Na rit a bin ich für seine freundliche Mithilfe zu Dank verpflichtet.

\section{Literatur.}

(1) Lund, E. J.; J. Exp. Zool., 1928, 51, 291.

(2) Willia m, M. M. D. u. Sheard, C.; Protoplasma., 1923, 15, 396.

(3) Taylor, A. B.; J. Cell. u. Comp. Physiol., 1935, 7, 1.

(4) Francis, W. L.; J. Exp. Biol., 1934, 11, 35.

(5) Ponder, E. u. Macleod, J.; J. of Gen. Physiol., 1937, 20, 433.

(6) Huf, E.; Pflügers Arch., 1935, 235, 655.

(7) Motokawa, K., Narita, T. u. Endo, N. ; Jap. J. Med., Sci, III Bioph., 1943, 9, 181.

(8) Motokawa, K., Narita, T. u. Endo, N.; Tohoku J. Exp. Med., 1943, 48.

(9) Endo, N.; Tohoku J. Exp. Med. 1943, 45, 218.

(10) Lund, E. J.; J. Exp. Zool., 1926, 44, 383.

(11) Narita, T.; Jap. J. Med., Sci., III Bioph., 1941, 7, 231.

(12) Adolph, E. F.; J. Exp. Zool., 1929, 53, 313.

(13) Huf, E. ; Bioch. Z., 1936, 288, 116.

(14) Huf, E. ; Pfügers Arch., 1938, 240, 573. 
(15) Motokawa, K.; Jap. J. Med., Sci., III Bioph., 1938, 5, 67.

(16) Beutner, R. u. Lozner, J., Protoplasma (Berl), 1933, 19, 370.

(17) Beutner, R.; Arch. exp. Zellforsch., 1934, 15, 217.

(18) Stern, K.; Pflanzen-Thermodynamik (Berlin), 1933, 346. 